\title{
Transient effects during sputtering of a-C:H surfaces by nitrogen ions
}

\author{
K. Dobes ${ }^{\mathrm{a}}$, P. Naderer ${ }^{\mathrm{a}}$, C. Hopf ${ }^{\mathrm{b}}$, T. Schwarz-Selinger ${ }^{\mathrm{b}}$, F. Aumayr ${ }^{\mathrm{a}, *}$ \\ anstitute of Applied Physics, TU Wien, Association EURATOM-ÖAW, 1040 Vienna, Austria \\ ${ }^{\mathrm{b}}$ Max-Planck-Institut für Plasmaphysik, EURATOM Association, Boltzmannstr. 2, 85748 Garching, Germany
}

\begin{abstract}
Sputtering of polymer-like amorphous hydrogenated carbon (a-C:H) thin films by $0.5-1 \mathrm{keV} \mathrm{N}_{2}^{+}$molecular ions has been studied in situ and real-time using a highly sensitive quartz crystal microbalance technique. During bombardment of a fresh, plasma-deposited a-C:H layer with nitrogen ions the measured sputtering yield decreases exponentially with ion fluence until a steady state value is reached at a fluence of typically about $3.5 \times 10^{15} \mathrm{~N}_{2}^{+}$ions per $\mathrm{cm}^{2}$. A chemical sputtering mechanism has to be considered in addition to physical sputtering to explain the observed steady state sputtering values. Simulations based on the code TRIDYN, which take into account a change of surface composition due to implantation and erosion, are performed to understand the transient development of sputtering yields.
\end{abstract}

Keywords: Ion-surface interaction, sputtering, amorphous carbon films (a-C:H), chemical erosion, chemical sputtering, codeposition

Published in Nuclear Instruments and Methods in Physics Research B: Received 11 August 2011

Available online 20 January 2012

Nucl. Instrum. Meth. B 286 (2012) 20-24

\section{INTRODUCTION}

In present fusion experiments carbon is still often used as first wall material because of its advantageous thermal properties. Carbon as a plasma-facing component, however, poses a serious problem for future fusion devices. Co-deposition of eroded carbon with hydrogen isotopes leads to formation of hydrogenated amorphous carbon $(\mathrm{a}-\mathrm{C}: \mathrm{H})$ layers which contain tritium. As a consequence a tritium inventory within the fusion vessel builds up over time and under reactor-like conditions is expected to rapidly exceed required safety limits [1].

Nitrogen seeding is presently used in fusion devices to reduce the local power load on highly exposed surfaces by enhanced radiative cooling [2]. This is why the interaction of nitrogen ions with a-C:H thin films is of considerable interest. It has recently been shown $[3,4]$ that a-C:H layers bombarded by molecular nitrogen $\mathrm{N}_{2}^{+}$ions exhibit an erosion rate which is enhanced by the chemical reactivity of the nitrogen projectiles especially at ion energies below the threshold for physical sputtering. By this, apart from radiatively cooling the edge plasma, the use of nitrogen as a seeding gas could also have a positive effect on the tritium inventory by eroding re-deposited a-C:T layers within a fusion device.

Hopf et al. [5] were able to model the energy dependence of this so-called chemical sputtering process on the basis of the following microscopic picture: when penetrating the a$\mathrm{C}: \mathrm{H}$ layer, energetic ions break $\mathrm{C}-\mathrm{C}$ bonds, which are eventually passivated by consecutive projectiles. In the case of chemically reactive projectiles, this leads to the formation of volatile species, which can diffuse to the surface and desorb there. Hopf et al. [5] showed that the chemical sputtering yield is thus proportional to the bond breaking density, i. e. the number of carbon displacement events per depth interval $y_{\mathrm{bb}}(x, E)$, and the ion implantation depth profile $n(x)$. Ac-

*Email address: aumayr@iap.tuwien.ac.at cording to the above mechanism chemical sputtering requires the presence of chemically reactive species such as hydrogen or (in our case) nitrogen, while physical sputtering does not. Since chemical sputtering only takes place at sites where reactive atoms (i.e. hydrogen or nitrogen) are present, the corresponding yield is thus assumed to be proportional to their respective atomic fractions within the target, while physical sputtering is proportional to the complementary fraction, i.e. the number of 'pure' carbon sites, where no reactive species is available in the vicinity. By this the authors in [5] were furthermore able to describe the dependence of the sputtering yield on the incoming ion flux.

In this work we report on investigations of the total sputtering yield of polymer-like, 'soft' a-C:H layers with a hydrogen content of $50 \%$ under $\mathrm{N}_{2}^{+}$ion bombardment using a highly sensitive quartz crystal microbalance technique (Section 2). Due to this high hydrogen content, 'soft' a-C:H layers exhibit the characteristics of an insulating layer, while 'hard' a-C:H films contain less hydrogen (typically $30 \%$ ) and are better conducting surfaces. When exposing a fresh a-C:H layer to a nitrogen ion beam we find, that the erosion yield at first exponentially decreases with ion fluence until a steady state value is reached after applying some $10^{15} \mathrm{~N}_{2}^{+}$to the surface (Section 3). We use the above-described chemical sputtering model by Hopf et al. [5] to explain our measured steady state sputtering yields and present simulation calculations based on the code TRIDYN to understand the transient development of sputtering yields (Section 4).

\section{EXPERIMENTAL SETUP}

Total sputtering yields of 'soft' a-C:H layers under nitrogen bombardment are investigated at TU Wien under controlled laboratory conditions with a self-built quartz crystal microbalance (QCM) setup (Fig. 1). The mass sensitive part of the QCM is a stress compensated (SC) cut quartz crystal driven at its thickness shear mode with a resonance fre- 


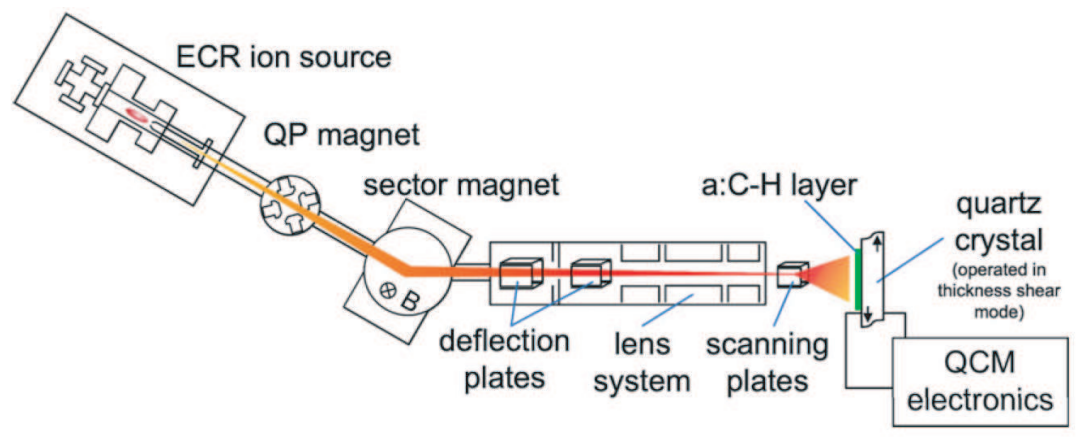

FIG. 1: Schematics of the experimental setup (not to scale).

quency of about $6 \mathrm{MHz}$. The QCM is operated at a temperature of $460 \mathrm{~K}$, where temperature changes induced by power deposition of the impinging ions have a minimal effect on the frequency. Sophisticated electronics permits the detection of mass changes of as small as $10^{-2}$ a-C:H monolayers per second. A detailed description of the QCM setup can be found elsewhere [68].

In an ECR methane plasma discharge a 'soft', polymer-like a-C:H layer with a hydrogen content of $50 \%$ is deposited onto one of the polished gold electrodes of the quartz crystal [9]. The film growth and characteristics of the layer was monitored by in situ ellipsometry. The thickness of the films was determined to be about $370 \mathrm{~nm}$. The sample is then transferred ex vacuo to an UHV experimental chamber, which is connected to the ECR ion source facility at Vienna University of Technology. It is mounted onto a sample holder, which is equipped with a Faraday cup for ion current determination and which is furthermore rotatable and moveable in $x y z$ direction.

Molecular nitrogen ions are produced in a $14.5 \mathrm{GHz}$ ECR ion source [10]. After extraction they are mass-over-charge selected with a sector magnet and focused onto the sample holder by means of an electrostatic lens system (Fig. 1). A pair of deflection plates right in front of the target is used to uniformly irradiate the active area of the quartz crystal by rapidly scanning the ion beam over the surface. By this, the mechanical stress imposed onto the quartz by a non-uniform erosion of the target film can be minimized and an accurate determination of the effective ion current density is possible. The Faraday-cup is used to scan the beam profile and monitor the ion current in between individual measurements.

The total mass change of the target film is determined from the eigenfrequency change of the quartz crystal $\Delta f / f=$ $-\Delta m / m$ [11]. From this the absolute mass loss per incident ion is determined by using the relation

$$
Y=\frac{\left(\rho_{\mathrm{Q}} \ell_{\mathrm{Q}}\right) / f}{I /\left(e_{0} A\right)} \frac{\Delta f}{\Delta t}
$$

where $\rho_{\mathrm{Q}}$ is the quartz' density, $\ell_{\mathrm{Q}}$ its thickness and $f$ its resonance frequency and $I /\left(e_{0} A\right)$ is the ion current density per unit area impinging on the quartz crystal.

In order to accurately determine the mass change under ion irradiation, the frequency change of the quartz crystal induced by the $\mathrm{N}_{2}^{+}$beam is corrected by the quartz crystal's intrinsic frequency drift at rest. For this purpose, measurements with ion beam are periodically alternated by 'beam-off' periods (for details see [6]). The sputtering yield, which in our experiment is measured in atomic mass units (amu) per incident projectile, is derived from the slope of a fit to the frequency change-curve. To investigate the evolution of a non-constant sputtering yield, consecutive measurements can be re-joined to a single curve. We note, that the QCM technique is only able to detect total mass changes, i.e. "net erosion" or "net deposition", but does not deliver information on actually sputtered species.

\section{EXPERIMENTAL RESULTS}

Fig. 2 shows, how the QCM frequency measured for impact of $1 \mathrm{keV} \mathrm{N} \mathrm{N}_{2}^{+}$ions (i.e. $500 \mathrm{eV}$ per $\mathrm{N}$ atom) changes its slope with increasing ion fluence, indicating a significant change in erosion yield during the course of the measurements. The corresponding evolution of the sputtering yield as a function of the ion fluence is shown in Fig. 3. The measurements started with a virgin, i. e. freshly deposited 'soft' a-C:H layer. Fluences and sputtering yields are given per incident nitrogen atom (not $\mathrm{N}_{2}$ molecule). At the beginning of the ion irradiation an elevated sputtering yield is observed that decreases exponentially with fluence to approximately $1 / 5$ of its initial value. After approximately $7 \times 10^{15} \mathrm{~N}$ atoms per $\mathrm{cm}^{2}$ have been applied to the surface a steady state value of the sputtering yield is finally reached (Fig. 3). At this fluence approximately $4 \mathrm{~nm}$ of target material have been removed, as can be estimated from the observed sputtering yield and with a target density of $1.1 \mathrm{~g} / \mathrm{cm}^{3}$ [3]. A similar behavior was also found for other impact energies ( $500 \mathrm{eV}$ and $700 \mathrm{eV} \mathrm{N}_{2}^{+}$projectiles), always using a fresh a-C:H film of course.

Fig. 4 summarizes the steady state sputtering yields obtained at the end of each measurement series. For comparison data by Jacob et al. [3] for bombardment of 'hard' a-C:H layers are shown. These data were obtained for a-C:H layers of lower hydrogen content $(30 \%)$, than in our case. Mind also, that in [3] the number of eroded carbon atoms per incident $\mathrm{N}$ atom is determined as a direct consequence of the measurement technique. With our technique on the other hand, the 


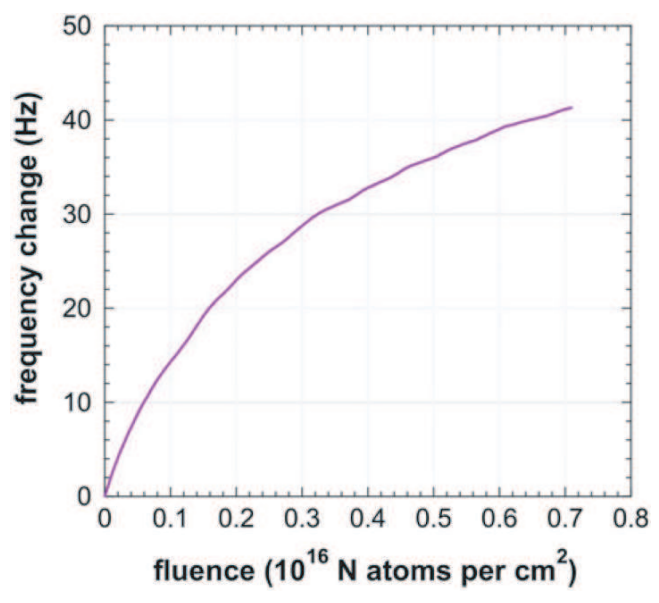

FIG. 2: Evolution of the quartz frequency during $1 \mathrm{keV} \mathrm{N}_{2}^{+}$ion bombardment of a 'soft' a-C:H surface as a function of applied ion fluence. At the beginning of the irradiation a strongly non-linear change of the resonance frequency of the quartz is observed.

total mass removal (including hydrogen, nitrogen and heavier compounds such as different kind of hydrocarbons $\mathrm{C}_{x} \mathrm{H}_{y}$ and other volatile molecules) is obtained. Both differences might explain the deviations between our data and those of Ref. [3].

The code TRIDYN [12] has been used to perform simulations of the interaction of nitrogen with a-C:H (see also Section 4). In these simulations the molecular nitrogen ion is treated as two separate atomic nitrogen projectiles at the same velocity or equivalently at the same specific energy (i.e. half the impact energy). When just taking into account physical sputtering alone, the TRIDYN code delivers steady state sputtering yields, which are shown in Fig. 4 by a green dashed line. Evidently it is essential to also include the chemical sputtering yield mechanism proposed by Hopf et al. [5] in the TRIDYN calculation (see red curve in Fig. 4) in order to adequately reproduce the observed steady state sputtering yields (see also Section 4).

\section{MODELING}

In the following a model shall be introduced, aiming to qualitatively describe both the transient as well as the steady state sputtering behavior observed in our experiments. It is based on the chemical sputtering model proposed by Hopf et al. [5]. Within the framework of this model, some simplified physical and chemical sputtering processes describe the interaction between target and projectile. They are used to establish a set of rate equations, which are able to reproduce the measured data. The model presented here, shall make the experimental results plausible and give a qualitative picture of the involved interaction processes. For a more detailed and complete description of the system, an atomistic- level simulation by means of e.g. molecular dynamics would be more favorable [13].

Within our simplified picture, chemical sputtering is de-

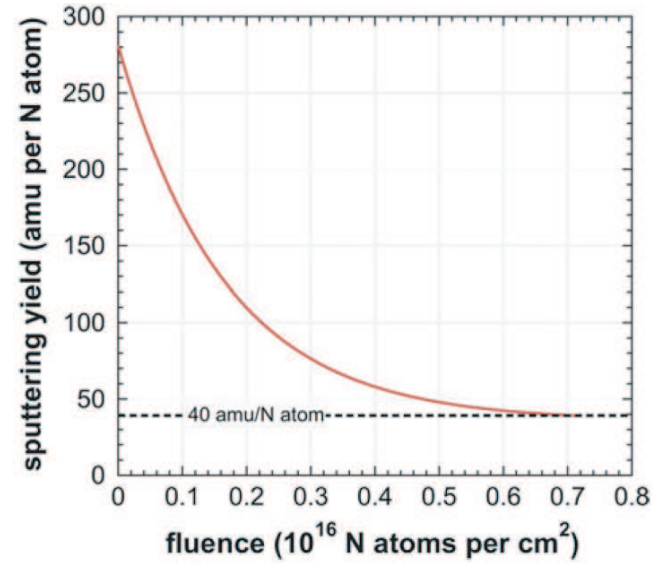

FIG. 3: Total sputtering yield of the a-C:H surface under bombardment by $1 \mathrm{keV} \mathrm{N}_{2}^{+}$ions (in atomic mass units per incident $\mathrm{N}$ atom) as a function of ion fluence. Data are evaluated from the slope of the frequency curve shown in Fig. 2.

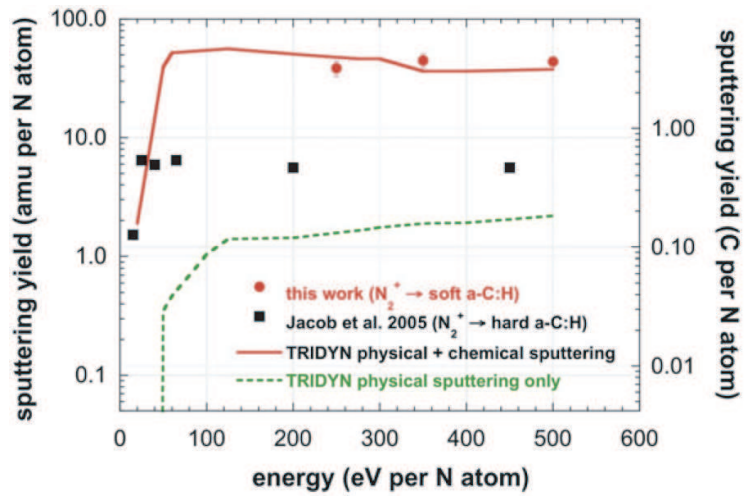

FIG. 4: Comparison of the obtained steady state sputtering yields (full red circles) for impact of $\mathrm{N}_{2}^{+}$ions on 'soft' a-C:H films with previous data and TRIDYN calculations. QCM results are given in atomic mass units per incident $\mathrm{N}$ atom and plotted vs. specific energy in $\mathrm{eV}$ per $\mathrm{N}$ atom. Black squares show experimental data by Jacob et al. [3] for bombardment of 'hard' a-C:H surfaces. Their sputtering yields are given in $\mathrm{C}$ atoms per incident $\mathrm{N}$ atom. Steady state sputtering yields calculated by TRIDYN [12] using the model for chemical sputtering by Hopf et al. [5] (full red curve) and are compared to calculations, which consider physical sputtering only (broken green curve). (For interpretation of the references to colour in this figure legend, the reader is referred to the web version of this article.)

scribed as a result of the following process: Impinging nitrogen ions (energy $E$ ) break $\mathrm{C}-\mathrm{C}$ bonds with a bond breaking yield density of $y_{\mathrm{bb}}(x, E)$, which is equal to the number of events along the ion penetration path (along the $x$ coordinate), in which the recoil atom in a binary collision receives an energy bigger than the bond breaking energy $E_{\mathrm{bb}}$. Thirteen electronvolt was found to be a reasonable value for $E_{\mathrm{bb}}$ [4] in order to describe the interaction of nitrogen with a-C:H. Subsequent projectiles then passivate the resulting dangling bonds forming volatile species. This process is dependent on 
the availability of nitrogen ions, which in turn is determined from the ion implantation depth profile $n(x, E)$. The volatile species diffuse to the surface with a probability of $\mathrm{e}^{-x / \lambda}$ and desorb there. Herein the decay length $\lambda$ is set to $0.4 \mathrm{~nm}$ [5]. The total sputtering yield $Y_{\text {tot }}$ will then be given by [5]

$$
\begin{aligned}
Y_{\mathrm{tot}}(E) & =Y_{\text {phys }}(E)+Y_{\mathrm{cs}}(E) \\
& =Y_{\text {phys }}(E)+a \int y_{\mathrm{bb}}(x, E) n(x, E) \mathrm{d} x
\end{aligned}
$$

with $a$ being a proportionality factor and $Y_{\text {phys }}$ and $Y_{\mathrm{cs}}$ denoting the physical and chemical sputtering yield, respectively. Chemical sputtering only takes place at sites where either $\mathrm{H}$ or $\mathrm{N}$ is present, while $\mathrm{C}$ sites are assumed to be physically sputtered. The proportionality factor $a$ takes into account uncertainties such as how many $\mathrm{N}$ or $\mathrm{H}$ are on average needed for one chemical sputtering event. The surface can be subdivided into concentrations $\Theta_{\mathrm{H}}$ and $\Theta_{\mathrm{N}}$ of sites where either $\mathrm{H}$ or $\mathrm{N}$ is present in the vicinity of $\mathrm{C}$ and a complementary fraction $\Theta_{\mathrm{C}}=1-\Theta_{\mathrm{H}}-\Theta_{\mathrm{N}}$ of 'pure' $\mathrm{C}$ sites. $\mathrm{H}$ and $\mathrm{N}$ sites are then chemically sputtered with a maximum yield of $Y_{\mathrm{cs}}^{\max }=Y_{\mathrm{cs}}\left(\Theta^{\mathrm{H}}+\Theta^{\mathrm{N}}=1\right)$. Here, for simplicity, it is assumed that the chemical sputtering yield for nitrogen and hydrogen is equal. $\mathrm{C}$ sites on the other hand are physically sputtered with a maximum yield of $Y_{\text {phys }}^{\max }=Y_{\text {phys }} \cdot\left(\Theta_{\mathrm{C}}=1\right) . Y_{\text {phys }}^{\max }$ and $Y_{\mathrm{cs}}^{\max }$ are regarded as limiting cases for zero and maximum $\mathrm{H}$ or $\mathrm{N}$ concentration in the target. The total sputtering yield can then also be written as

$$
Y_{\text {tot }}=Y_{\mathrm{cs}}^{\max } \cdot\left(\Theta_{1}^{\mathrm{H}}+\Theta_{1}^{\mathrm{N}}\right)+Y_{\text {phys }}^{\max } \cdot\left(1-\Theta_{1}^{\mathrm{H}}-\Theta_{1}^{\mathrm{N}}\right) .
$$

To describe the observed steady state sputtering yields from Fig. 4, in a first step $Y_{\text {tot }}$ is calculated from Eq. (2) with TRYDIN for the case of $1 \mathrm{keV} \mathrm{N}_{2}^{+}$ions, which are assumed to be equal to separate atomic $\mathrm{N}$ projectiles with half the energy. As initial target we use an a-C:H film with a fraction of $\mathrm{H} / \mathrm{C}=1$ and a density of $1.1 \mathrm{~g} / \mathrm{cm}^{3}$. The surface binding energy for carbon and hydrogen ions is set to $2.8 \mathrm{eV}$, the surface binding energy for nitrogen is taken to be $1.9 \mathrm{eV}$ [3]. Both the physical as well as the chemical sputtering yield are determined at a fluence of $7 \times 10^{15} \mathrm{~N}$ atoms per $\mathrm{cm}^{2}$, which corresponds to steady state conditions in our measurements. The resulting TRIDYN curve depicted in Fig. 4 is obtained using a proportionally factor of $a=5$ in Eq. (2). As seen from Fig. 4 good agreement with our experimental data is obtained for all investigated ion energies.

The observed dependence of the sputtering yield on ion fluence (Fig. 3) reflects the modification (change of composition) of the a-C:H layer during ion impact. Modeling of these processes tended to be more difficult. In a first step the evolution of the atomic fractions of $\mathrm{H}, \mathrm{N}$ and $\mathrm{C}$ under ion bombardment were calculated from a balance of different processes: Hydrogen and nitrogen are removed from the surface by either ion-induced depletion due to momentum transfer with a yield of $Y_{\mathrm{depl}}$ or by chemical sputtering $Y_{\mathrm{cs}}$. Impinging nitrogen atoms are furthermore implanted at $\mathrm{C}-\mathrm{C}$ sites with an implantation yield of $Y_{\mathrm{imp}}$. As the surface is eroded, bulk material with atomic concentrations different from those in the top layer emerges into the ion range. To account for this, we slice the target into four $2 \mathrm{~nm}$ thick sub-layers and an underlying bulk with fixed atomic concentrations corresponding to the initial conditions. We calculate the distinct yields for the above-mentioned processes for each layer separately with TRIDYN at a fluence of $7 \times 10^{15} \mathrm{~N}$ atoms per $\mathrm{cm}^{2}$. By using TRIDYN instead of TRIM, compositional changes of the surface as a result of projectile implantation are taken into account too. Hereby $Y_{\text {depl }}$ is determined from the number of displaced atoms in a collision cascade within such a layer minus the number of recoil atoms, which come to rest in this layer. $Y_{\text {imp }}$ is derived from the implantation depth profile $n(x) . Y_{\mathrm{cs}}$ is determined from Eq. (2). The evolution of the surface concentrations in the four sub-layers $i=1-4$ is then given by the following rate equations:

$$
\begin{aligned}
n_{0} \frac{\mathrm{d} \Theta_{i}^{\mathrm{H}}}{\mathrm{d} t}= & -j_{\mathrm{N}}\left(Y_{\mathrm{depl}}^{\mathrm{H}}+Y_{\mathrm{cs}}^{\mathrm{H}}\right) \Theta_{i}^{\mathrm{H}}+j_{\mathrm{N}} Y_{\mathrm{tot}} \Theta_{i+1}^{\mathrm{H}} \\
n_{0} \frac{\mathrm{d} \Theta_{i}^{\mathrm{N}}}{\mathrm{d} t}= & -j_{\mathrm{N}}\left(Y_{\mathrm{depl}}^{\mathrm{H}}+Y_{\mathrm{cs}}^{\mathrm{H}}\right) \Theta_{i}^{\mathrm{N}} \\
& +j_{\mathrm{N}}\left(1-\Theta_{i}^{\mathrm{N}}-\Theta_{i}^{\mathrm{H}}\right) Y_{\mathrm{imp}}^{\mathrm{N}}+j_{\mathrm{N}} Y_{\mathrm{tot}} \Theta_{i+1}^{\mathrm{N}} .
\end{aligned}
$$

With the term $j_{\mathrm{N}} Y_{\text {tot }} \Theta_{i}^{\mathrm{N}}$ the exchange of a particular sub-layer with its individual subjacent layer as a consequence of surface erosion is included. The composition of the undermost sublayer $i=4$ corresponds to the initial bulk concentrations.

In Fig. 5(a) the resulting concentrations of $\mathrm{C}, \mathrm{H}$ and $\mathrm{N}$ in the topmost sub-layer are depicted as a function of ion fluence. From the rate equations (4) and (5), one finds that under ion bombardment a nitrogen concentration builds up within the surface, while the hydrogen concentration decreases with impinging ion fluence.

From the evolution of the surface composition under ion bombardment, the number of removed particles per incident nitrogen atom due to the respective sputtering processes in Eq. (3) can be calculated. For a comparison with our experimental data from Fig. 4, however, a total mass removal (in amu per nitrogen atom) has to be deduced from these yields, since this is the quantity accessible with our experimental technique. In doing so, somewhat arbitrary assumptions on the masses of the volatile compounds formed and sub-sequentially desorbed from the surface in the chemical sputtering processes $Y_{\mathrm{cs}}^{\mathrm{H}}$ and $Y_{\mathrm{cs}}^{\mathrm{N}}$ have to be made. In an attempt of bringing the measured total sputtering yields from Fig. 4 into agreement with $Y_{\text {tot }}$ as obtained from Eq. (3), we used typical masses for molecules as reported in the literature such as $\mathrm{CN}, \mathrm{HCN}, \mathrm{C}_{2} \mathrm{~N}_{2}$ [14] and different kinds of hydrocarbons $\mathrm{C}_{x} \mathrm{H}_{y}$, respectively. However, regardless of the applied molecule mass, the evolution of the total sputtering yield with ion fluence was inconsistent with the measured data. Especially our model failed to describe the substantial difference between the experimentally observed initial sputtering yield to the yield under steady state conditions.

When investigating sputtering rates of different polymers under ion irradiation with a QCM technique, Zekonyte et al. [15] observed a dynamic change in the total mass removal with ion fluence very similar to the one found in our experiments. In their investigations these authors found out that 

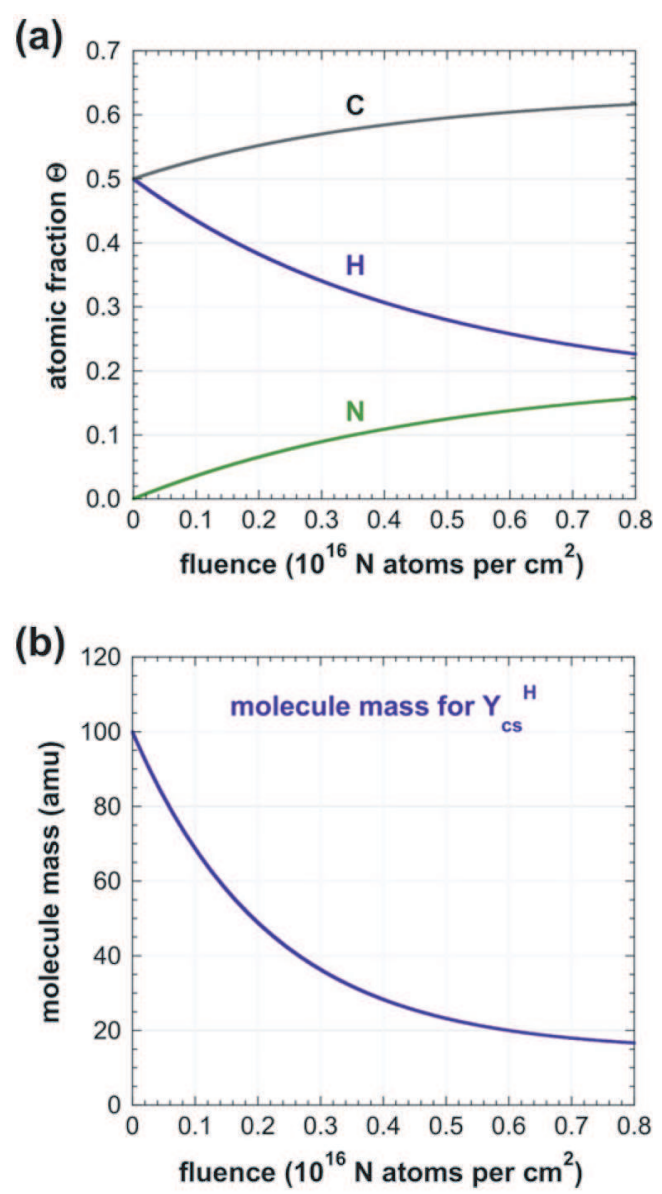

FIG. 5: (a) Fluence dependence of the atomic fractions of $\mathrm{C}$ (black curve), $\mathrm{H}$ (blue curve) and $\mathrm{N}$ (green curve) in the a-C:H surface under $\mathrm{N}$ bombardment as obtained from the set of rate equations (cf. Eqs. (4) and (5)) describing the interaction of the projectiles with the surface. (b) Evolution of the molecular mass of the $\mathrm{C}_{x} \mathrm{H}_{y}$ species chemically sputtered at $\mathrm{H}$ sites, which had to be assumed to correctly model the evolution of the total sputtering yield with ion fluence. (For interpretation of the references to colour in this figure legend, the reader is referred to the web version of this article.)

the observed transient effect in the sputtering yield is linked to a chemical modification of the target film due to ion bombardment. The decrease in sputtering yield with ion fluence was additionally enhanced by a substantial reduction of higher molecular weight fragments of the sputtered species (as confirmed by secondary ion mass spectrometry measurements [16]).

The formation of a modified surface layer of an a-C:H film under ion bombardment was also found by von Keudell et al. [17]. These authors observed a densification of the target film and attributed this to the formation of new $\mathrm{C}-\mathrm{C}$ bonds as a result of ion bombardment.

The findings in $[15,17]$ strongly suggest that assuming a constant mass for the chemically sputtered species constitutes an oversimplification of the problem and that it is necessary to introduce a change of the eroded molecule mass with imping-

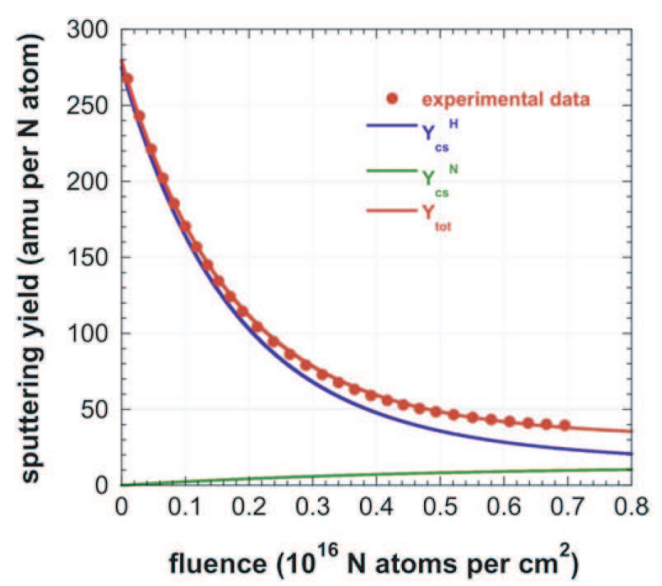

FIG. 6: Comparison of the modeled total sputtering yield (red solid line) with results obtained from the experiment (red circles). The evolution of $Y_{\mathrm{cs}}^{\mathrm{H}}$ (blue solid line) was obtained assuming that the sputtered molecule masses change with ion fluence (cf. Fig. 5(b)), while for $Y_{\mathrm{cs}}^{\mathrm{N}}$ (green solid line) the sputtered molecules were assumed to be of constant mass. Note that for determining the $Y_{\text {tot }}$, a constant physical sputtering yield (cf. Fig. 4) is also included, which is not depicted here. (For interpretation of the references to colour in this figure legend, the reader is referred to the web version of this article.)

ing ion fluence to our model, to describe the transient sputtering yield observed in our experiment appropriately.

Since the above-described processes are governed by a modification of the chemical structure of the target film, we suggest accounting for the change of the molecule mass by varying the mass of the eroded $\mathrm{C}_{x} \mathrm{H}_{y}$ molecules only. This, of course, will strongly affect the evolution of $Y_{\mathrm{cs}}^{\mathrm{H}}$ and consequently also of $Y_{\text {tot. }}$. We therefore assumed that the mass of the $\mathrm{C}_{x} \mathrm{H}_{y}$ species decreases with ion fluence and eventually reaches a steady state value. The function dependence was adapted in a way to optimally reproduce the yield evolution in Fig. 3. Such found evolution of the molecular mass of the eroded $\mathrm{C}_{x} \mathrm{H}_{y}$ species is depicted in Fig. 5(b). Initially molecule masses around $100 \mathrm{amu}$ are eroded per incident nitrogen atom. This value decreases exponentially until under steady state conditions molecules with a mean mass of $15 \mathrm{amu}$ per incident $\mathrm{N}$ atom are sputtered. With this assumption the modeled evolution of the total sputtering yield with ion fluence is in very good agreement with our experimentally findings. In Fig. 6 both the experimental results (red dots) as well as the modeled evolution of the total sputtering yield (red line) are depicted. In addition the underlying evolutions of $Y_{\mathrm{cs}}^{\mathrm{H}}$ (blue line) and $Y_{\mathrm{cs}}^{\mathrm{N}}$ (green line) are also shown.

\section{CONCLUSIONS}

We have investigated the sputtering of 'soft', polymer-like a-C:H layers under $\mathrm{N}_{2}^{+}$bombardment at ion energies up to $1 \mathrm{keV}$. We have found that the total sputtering yield observed, at first decreases strongly with ion fluence and reaches a steady state value after some $10^{15} \mathrm{~N}_{2}^{+}$ions per $\mathrm{cm}^{2}$ have been 
applied to the surface. With the help of TRIDYN simulations and a model, which aside from physical sputtering also describes chemical sputtering [5], the observed steady state sputtering yields as a function of impact energy could be reproduced nicely.

In order to understand the transient behavior of the sputtering yield, a set of rate equations for the atomic composition of the target was considered, which takes into account various interaction processes between projectile and target. A change in surface composition due to the sputtering process alone is, however, not able to explain the strong decrease of the observed sputtering yield with ion fluence. Good agreement between model and experiment could only be obtained by assuming that the average mass of the hydrocarbon molecules eroded in the chemical sputtering processes also decreases with ion fluence.

\section{ACKNOWLEDGEMENTS}

The authors are grateful to Prof. M. Schmid (IAP,TU Wien) for his continued support with the QCM electronics. Financial support has been provided by KKKÖ (commission for the coordination of fusion research in Austria) and Austrian FWF. This work, supported by the European Commission under the Contract of Association between EURATOM and ÖAW, was carried out within the framework of the European Fusion Development Agreement (EFDA). The views and opinions expressed herein do not necessarily reflect those of the European Commission.

\section{References}

[1] G. Federici et al., Nucl. Fusion 4 (2001) 1967.

[2] J. Rapp et al., J. Nucl. Mat. 337339 (2005) 826.

[3] W. Jacob, C. Hopf, M. Schlüter, Appl. Phys. Lett. 86 (2005) 204103

[4] M. Schlüter, C. Hopf, W. Jacob, New J. Phys. 10 (2008) 053037.

[5] C. Hopf, A. von Keudell, W. Jacob, J. Appl. Phys. 94 (2003) 2373.

[6] G. Hayderer, M. Schmid, P. Varga, HP. Winter, F. Aumayr, Rev. Sci. Instrum. 70 (1999) 3696.

[7] G. Hayderer et al., Phys. Rev. Lett. 83 (1999) 3948.

[8] A. Golczewski, K. Dobes, G. Wachter, M. Schmid, F. Aumayr, Nucl. Instr. Meth. B 267 (2009) 695.

[9] T. Schwarz-Selinger, A. von Keudell, W. Jacob, J. Appl. Phys. 86 (1999) 3988.

[10] E. Galutschek, R. Trassl, E. Salzborn, F. Aumayr, HP. Winter, J. Phys. Conf. Ser. 58 (2007) 395.

[11] G. Sauerbrey, Z. Phys. 155 (1959) 206.

[12] W. Möller, W. Eckstein, J.P. Biersack, Comp. Phys. Commun. 51 (1988) 355

[13] P. N. Maya, U. von Toussaint, C. Hopf, New J. Phys. 10 (2008) 023002 .

[14] P. Hammer, W. Gissler, Diamond Relat. Mater. 5 (1996) 1152.

[15] J. Zekonyte, V. Zaporojtchenko, F. Faupel, Nucl. Instr. Meth. B 236 (2005) 241

[16] D. Briggs, A. B. Wooton, Surf. Interface Anal. 4 (1982) 109.

[17] A. von Keudell, W. Jacob, W. Fukarek, Appl. Phys. Lett. 66 (1995) 1322 . 